\title{
A call for benchmarking transposable element annotation methods
}

\author{
Douglas R. Hoen ${ }^{1,2^{*}}$, Glenn Hickey ${ }^{1,3}$, Guillaume Bourque ${ }^{4,5}$, Josep Casacuberta ${ }^{6}$, Richard Cordaux ${ }^{7}$, \\ Cédric Feschotte ${ }^{8}$, Anna-Sophie Fiston-Lavier ${ }^{9}$, Aurélie Hua-Van ${ }^{10}$, Robert Hubley ${ }^{11}$, Aurélie Kapusta ${ }^{8}$, \\ Emmanuelle Lerat ${ }^{12}$, Florian Maumus ${ }^{13}$, David D. Pollock ${ }^{14}$, Hadi Quesneville ${ }^{13}$, Arian Smit ${ }^{11}$, Travis J. Wheeler ${ }^{15}$, \\ Thomas E. Bureau ${ }^{2}$ and Mathieu Blanchette ${ }^{1,3^{*}}$
}

\begin{abstract}
DNA derived from transposable elements (TEs) constitutes large parts of the genomes of complex eukaryotes, with major impacts not only on genomic research but also on how organisms evolve and function. Although a variety of methods and tools have been developed to detect and annotate TEs, there are as yet no standard benchmarks - that is, no standard way to measure or compare their accuracy. This lack of accuracy assessment calls into question conclusions from a wide range of research that depends explicitly or implicitly on TE annotation. In the absence of standard benchmarks, toolmakers are impeded in improving their tools, annotators cannot properly assess which tools might best suit their needs, and downstream researchers cannot judge how accuracy limitations might impact their studies. We therefore propose that the TE research community create and adopt standard TE annotation benchmarks, and we call for other researchers to join the authors in making this long-overdue effort a success.
\end{abstract}

\section{Why does transposable element annotation matter, and why is it difficult?}

Transposable elements (TEs) are segments of DNA that self-replicate in a genome. DNA segments that originated from TE duplications may or may not remain transpositionally active but are herein referred to simply as TEs. TEs form vast families of interspersed repeats and constitute large parts of eukaryotic genomes, for example, over half of the human genome [1-3] and over four fifths of the maize genome [4]. The repetitive nature of TEs confounds many types of studies, such as gene prediction, variant calling (i.e., the identification of sequence variants such as SNPs or indels), RNA-Seq analysis, and genome alignment. Yet their mobility and repetitiveness also endow TEs with the capacity to contribute to diverse aspects of biology, from disease [5], to genome evolution [6-8], organismal development [9], and gene regulation [10]. In addition to dramatically affecting genome size,

\footnotetext{
* Correspondence: douglas.hoen@mcgill.ca; blanchem@cs.mcgill.ca 'School of Computer Science, McGill University, McConnell Engineering Bldg., Rm. 318, 3480 Rue University, Montréal, Québec H3A 0E9, Canada ${ }^{3}$ McGill Centre for Bioinformatics, McGill University, Montréal, Québec, Canada

Full list of author information is available at the end of the article
}

structure (e.g., chromatin organization), variation (e.g., copy-number variation), and chromosome maintenance (e.g., centromere and telomere maintenance) [11], TEs also provide the raw material for evolutionary innovation, such as the formation of new protein-coding genes [12, 13], non-coding RNAs [14-16], and transcription factor binding sites $[17,18]$. With the growing deluge of genomic data, it is becoming increasingly critical that researchers be able to accurately and automatically identify TEs in genomic sequences.

Accurately detecting and annotating TEs are difficult because of their great diversity, both within and among genomes. There are many types of TE $[19,20]$, which differ across multiple attributes, including transposition mechanism, TE structure, sequence, length, repetitiveness, and chromosomal distribution. Moreover, while recently inserted TEs have relatively low within-family variability, over time TE instances (specific copies) accumulate mutations and diverge, becoming ever more difficult to detect. Indeed, much of the DNA with as yet unknown origins in some genomes (e.g., human) might be highly decayed TE remnants $[2,8]$. Because of this great diversity TEs within and among genomes, the 
primary obstacles to accurately annotating TEs vary dramatically among genomes, which have different TE silencing systems and which have undergone different patterns of TE activity and turnover. For instance, in some genomes (e.g., human [1]) the majority of TEderived DNA is remnant of ancient bursts in the activity of just a few TE families; thus, annotation is mainly hampered by the high divergence of old and decayed TE copies, as well as extensive fragmentation of individual copies and the complex evolution of the TEs in the genome [6]. Other genomes (e.g., maize [4]) contain a large variety of recently active TEs; thus, defining and classifying the diverse families poses a considerable annotation challenge, as well as disentangling the complex and heterogeneous structures formed by clusters of TEs, such as internal deletions, nested insertions, and other rearrangements [21]. Furthermore, although libraries of known TE sequences are definitely useful, the TE families that are present in even closely related genomes may differ greatly [22], limiting the utility of such libraries in annotating newly sequenced genomes. Additional challenges to accurate annotation arise from multi-copy non-TE (host) gene families and segmental duplications, which in both cases mimic TEs because of their repetitiveness. Low complexity sequences and simple repeats may also be major sources of false positives [23]. Together, these issues pose considerable challenges to accurate, automated TE annotation.

Although the field of TE annotation may be broadly defined to include various activities, such as the identification and classification of TE families $[19,20]$, herein, we mainly discuss the detection and annotation of TE instances, particularly within assembled genomes, and the computational tools used to do so. A number of computational approaches and tools have been developed to identify TEs in assembled genomes. The two main approaches used currently are homology-based approaches, which use similarity to known TEs, and de novo approaches, which are typically based either on repetitiveness or on structural signatures (e.g., long terminal repeats or terminal inverted repeats) (reviewed in [24-26]). In addition, approaches are being developed to detect TEs using comparative genomics (e.g., insertion polymorphisms) [27] (Hickey et al., pers. comm.) or other properties such as the production of specific populations of small RNAs (e.g., siRNAs, piRNAs) [28]. However, to annotate assembled genomes, most researchers have implicitly adopted a de facto standard of tool use that incorporates just a fraction of available tools (Table 1), as follows: (i) Mask simple repeats (e.g., TRF [29]); (ii) Generate a library of ostensible TE sequences using repetitiveness-based tools (e.g., RepeatModeler, RepeatScout [30-32]), often augmented with one or more structure-based programs (e.g., LTR_FINDER [33],
LTR_STRUC [34], or MITE-Hunter [35]); (iii) Classify consensus sequences into families (e.g., RepeatModeler [30] or RepClass [36]); (iv) Combine with an existing library of TE consensus sequences (or models) (e.g., RepBase [37] or recently Dfam [3]); (v) Finally, align the TE consensus sequences (or models) to the genome (e.g., either RepeatMasker [38] or Censor [39] with dependencies on sequence similarity tools such as cross_match [40], BLAST [41, 42], or nhmmer [43]). Different annotators often use and combine the tools in different ways, using different settings and ad hoc results filtering, library merging, and manual steps. A few groups have developed more complete pipelines that combine a wider selection of tools in a consistent manner (e.g., REPET [44]). A growing number of tools also operate directly on unassembled short genomic reads [45-50]. Finally, there are a small number of groups using largely manual methods to refine the libraries generated by these automated pipelines to create high quality TE libraries (Table 1) [3, 37, 51].

\section{Why do we urgently need benchmarks?}

TE predictions made by various methods are often quite divergent, with different tools having different strengths and weaknesses, competencies, and complementarities $[8,24,52$, 53] (Fig. 1). Why then are so few tools commonly used? How optimal are the various combinations of tools that are used? Most importantly, how accurate are the TE annotations that are produced?

In related disciplines including genome assembly [54], multiple sequence alignment [55-57], variant calling [58, 59], and cancer genomics [60], standard benchmarks have been successfully employed to measure and improve the accuracy of computational tools and methodologies. For example, in the area of protein structure prediction, researchers have taken great efforts to tackle the benchmarking problem for over 20 years [61].

However, for TE annotation, there is currently no standard way to measure or compare the accuracy of particular methods or algorithms. In general, there is a tradeoff between increased rates of true vs. false positives, both between different tools and between different settings for any given tool, a tradeoff that should ideally be optimized for each study. For instance, a study attempting to describe reasonable upper bounds of TE contributions to genome size might benefit from increased sensitivity (at the cost of specificity), while a study attempting to identify high stringency TE-derived regulatory regions might benefit from the converse. Regardless of the approach chosen for a study-even if it is a de facto standard tool with default settings-the resultant tradeoff between false and true positives ought to be quantified and reported. However, the current state of TE annotation does not facilitate such distinctions, 
Table 1 Tools and databases used to annotate TEs in the genomes of multicellular eukaryotes published in 2014

\begin{tabular}{|c|c|c|c|c|c|c|c|c|c|c|c|c|c|c|c|}
\hline \multirow[t]{2}{*}{ Genome } & & \multicolumn{4}{|c|}{ Homology-based } & \multicolumn{6}{|l|}{ De novo } & \multirow{2}{*}{$\begin{array}{l}\text { Pipeline } \\
\text { REPET }\end{array}$} & \multirow[b]{2}{*}{ Other Databases } & \multirow[b]{2}{*}{ Other tools ${ }^{a}$} & \multirow[b]{2}{*}{ Ref. } \\
\hline & & Repbase & $\begin{array}{l}\text { Repeat } \\
\text { Masker }\end{array}$ & CENSOR & $\begin{array}{l}\text { Repeat } \\
\text { Protein } \\
\text { Mask }\end{array}$ & $\begin{array}{l}\text { Repeat } \\
\text { Modeler }\end{array}$ & $\begin{array}{l}\text { Repeat } \\
\text { Scout }\end{array}$ & PILER & LTR_FINDER & LTR_STRUC & $\begin{array}{l}\text { MITE- } \\
\text { Hunter }\end{array}$ & & & & \\
\hline $\begin{array}{l}\text { Phalaenopsis equestris } \\
\text { (tropical epiphytic orchid) }\end{array}$ & $\begin{array}{l}\text { Plant } \\
\text { (monocot) }\end{array}$ & $\checkmark$ & $\checkmark$ & & $\checkmark$ & & $\checkmark$ & $\checkmark$ & $\checkmark$ & $\checkmark$ & & & & & [81] \\
\hline $\begin{array}{l}\text { Cyprinus carpio } \\
\text { (common carp) }\end{array}$ & $\begin{array}{l}\text { Animal } \\
\text { (bony fish) }\end{array}$ & $\checkmark$ & $\checkmark$ & & & $\checkmark$ & & & & & & & & TEClass & [82] \\
\hline Esox lucius (northern pike) & $\begin{array}{l}\text { Animal } \\
\text { (bony fish) }\end{array}$ & $\checkmark$ & & $\checkmark$ & & & & & & & $\checkmark$ & & $\begin{array}{l}\text { Genbank, } \\
\text { UniprotKB/SwissProt }\end{array}$ & Custom & [83] \\
\hline $\begin{array}{l}\text { Oryza glaberrima } \\
\text { (African rice) }\end{array}$ & $\begin{array}{l}\text { Plant } \\
\text { (monocot) }\end{array}$ & $\checkmark$ & $\checkmark$ & & & & $\checkmark$ & & $\checkmark$ & & & & $\begin{array}{l}\text { MSU Repeats, custom } \\
\text { (rice-specific) }\end{array}$ & Custom & [84] \\
\hline $\begin{array}{l}\text { Callithrix jacchus } \\
\text { (common marmoset) }\end{array}$ & $\begin{array}{l}\text { Animal } \\
\text { (primate) }\end{array}$ & $\checkmark$ & $\checkmark$ & & & & & & & & & & & & [85] \\
\hline $\begin{array}{l}\text { Gossypium arboreum } \\
\text { (cultivated cotton) }\end{array}$ & Plant (dicot) & $\checkmark$ & $\checkmark$ & & $\checkmark$ & $\checkmark$ & $\checkmark$ & $\checkmark$ & $\checkmark$ & $\checkmark$ & & & & & [86] \\
\hline $\begin{array}{l}\text { Nicotiana tabacum } \\
\text { (common tobacco) }\end{array}$ & Plant (dicot) & & $\checkmark$ & & & & $\checkmark$ & & & & & & $\begin{array}{l}\text { TIGR, SGN } \\
\text { (Solanaceae-specific) }\end{array}$ & & [87] \\
\hline $\begin{array}{l}\text { Glossina morsitans } \\
\text { (tsetse fly) }\end{array}$ & $\begin{array}{l}\text { Animal } \\
\text { (insect) }\end{array}$ & & $\checkmark$ & & & $\checkmark$ & $\checkmark$ & & & & & $\checkmark$ & Genbank & $\begin{array}{l}\text { RECON, } \\
\text { TARGeT }\end{array}$ & [88] \\
\hline $\begin{array}{l}\text { Oncorhynchus mykiss } \\
\text { (rainbow trout) }\end{array}$ & $\begin{array}{l}\text { Animal } \\
\text { (bony fish) }\end{array}$ & $\checkmark$ & $\checkmark$ & $\checkmark$ & & $\checkmark$ & & & $\checkmark$ & & & & & $\begin{array}{l}\text { E-inverted, } \\
\text { Manual }\end{array}$ & [89] \\
\hline $\begin{array}{l}\text { Tetrao tetrix (black } \\
\text { grouse) }\end{array}$ & Animal (bird) & $\checkmark$ & $\checkmark$ & & & & & & & & & & & & [90] \\
\hline Pinus taeda (loblolly pine) & $\begin{array}{l}\text { Plant } \\
\text { (gymnosperm) }\end{array}$ & & $\checkmark$ & $\checkmark$ & & & & $\checkmark$ & & & $\checkmark$ & $\checkmark$ & $\begin{array}{l}\text { PIER } 2.0 \\
\text { (conifer-specific) }\end{array}$ & Custom & [91] \\
\hline $\begin{array}{l}\text { Spirodela polyrhiza } \\
\text { (duckweed) }\end{array}$ & $\begin{array}{l}\text { Plant } \\
\text { (monocot) }\end{array}$ & & $\checkmark$ & & & & & & & $\checkmark$ & & & $\begin{array}{l}\text { MipsREdat, MIPS } \\
\text { PlantsDB }\end{array}$ & Custom & [92] \\
\hline $\begin{array}{l}\text { Cynoglossus semilaevis } \\
\text { (half-smooth tongue sole) }\end{array}$ & $\begin{array}{l}\text { Animal } \\
\text { (flatfish) }\end{array}$ & & $\checkmark$ & & & & $\checkmark$ & $\checkmark$ & $\checkmark$ & & & & $\begin{array}{l}\text { RepBase (for } \\
\text { classification) }\end{array}$ & $\begin{array}{l}\text { E-inverted, } \\
\text { Custom }\end{array}$ & [93] \\
\hline $\begin{array}{l}\text { Capsicum annuum } \\
\text { L. and var. glabriusculum } \\
\text { (cult. and wild peppers) }\end{array}$ & Plant (dicot) & $\checkmark$ & $\checkmark$ & & $\checkmark$ & $\checkmark$ & & & $\checkmark$ & & & & MSU repeats & & [94] \\
\hline $\begin{array}{l}\text { Capsicum annuum cV. } \\
\text { CM334 (hot pepper) }\end{array}$ & Plant (dicot) & $\checkmark$ & $\checkmark$ & & & $\checkmark$ & & & & & & & & & [95] \\
\hline $\begin{array}{l}\text { Anopheles sinensis } \\
\text { (mosquito) }\end{array}$ & $\begin{array}{l}\text { Animal } \\
\text { (insect) }\end{array}$ & $\checkmark$ & $\checkmark$ & & & & & & & & & & $\begin{array}{l}\text { Efam } \\
\text { (mosquito-specific) }\end{array}$ & & [96] \\
\hline
\end{tabular}

${ }^{a}$ Not all tools used in building TE libraries are listed (e.g., UCLUST, MUSCLE) 


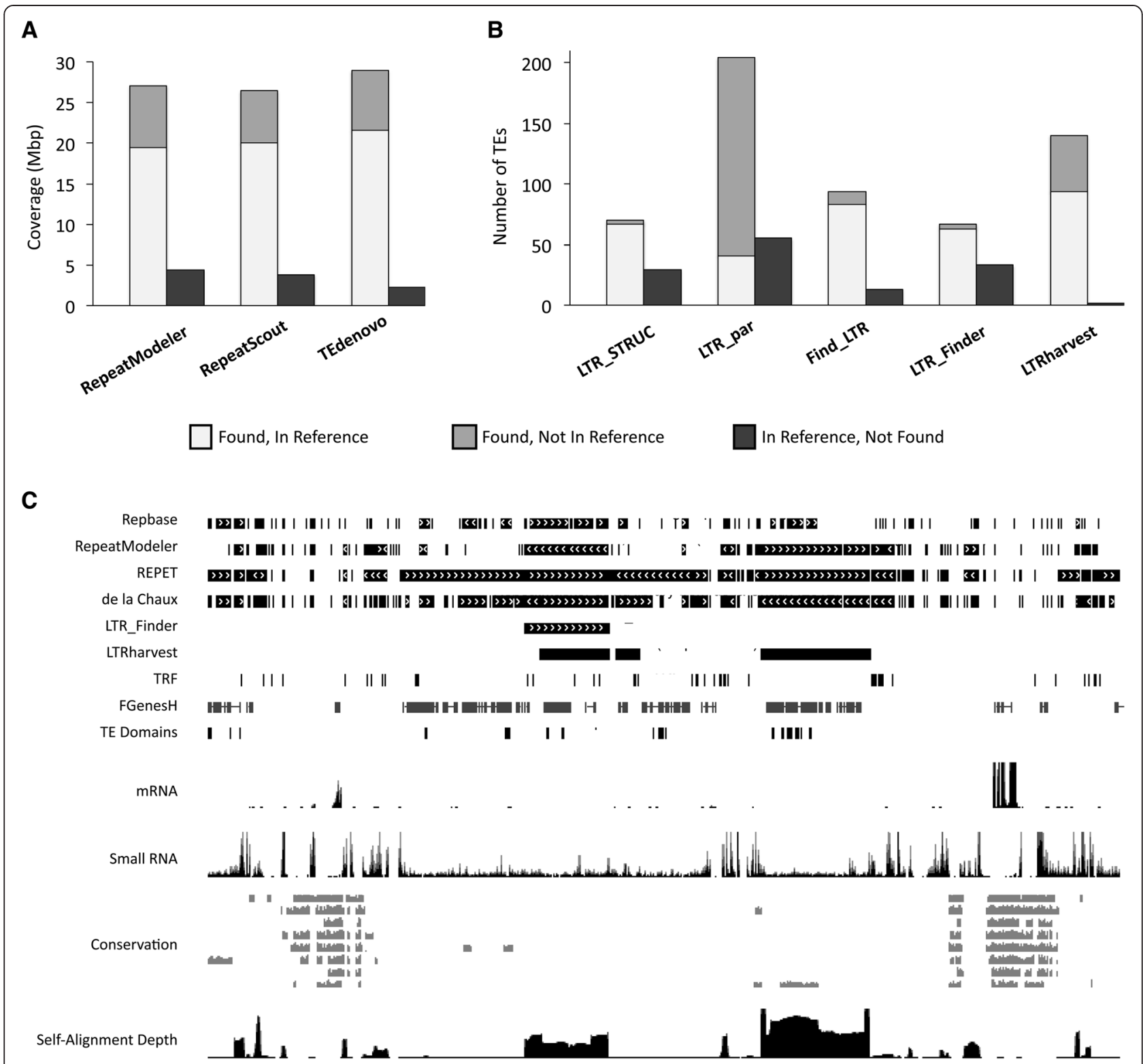

Fig. 1 Variation among TE annotation tools. a TE coverage in the Arabidopsis thaliana genome resulting from three commonly used repetitiveness-based de novo tools, compared to a reference set of TEs [8]. The total amount of TE coverage differs between the three, as does the fraction of the reference TEs that were found or missed and the amount of non-reference putative TEs. $\mathbf{b}$ Full-length LTR TEs in the Drosophila melanogaster $X$ chromosome found by five different LTR-specific de novo tools, compared to a reference set of TEs [24]. Similar to a but even more pronounced, the number of TEs found by the tools and their agreement with the reference set vary widely. c A 100-kbp segment of the Arabidopsis lyrata genome (scaffold_1:14,957,501-15,057,500) displayed on a custom UCSC genome browser [76, 77], illustrating differences among TE annotations resulting from several approaches, as well as additional genomic data useful in identifying bona fide TEs. From top to bottom, the tracks represent: RepeatMasker annotations using libraries from Repbase [37], RepeatModeler [30], REPET [44], or de la Chaux et al. [78]; full-length LTR TE predictions by LTR_Finder [33] or LTRharvest [79]; tandem repeat predictions by TRF [29]; gene models predictions by FGenesH [80]; a set of TE-specific domains [13]; mapped mRNA and small RNA short reads [77]; inter-species conservation (alignment percent identity plots) to other Brassicaceae species [77]; and genome self-alignment depth (generated with LASTZ)

especially for non-experts. Instead, it is left up to individual toolmakers, prospective tool users, or even downstream researchers to evaluate annotation accuracy. A few toolmakers with sufficient resources do invest the significant amount of effort required to assemble their own (often unpublished) test data sets and evaluate the accuracy of their tools. But for many toolmakers and most users, it is in practice too onerous to properly assess which methods, tools, and parameters may best suit their needs. The absence of standard benchmarks is thus 
an impediment to innovation because it reduces toolmakers' ability and motivation to develop new and more accurate tools or to improve the accuracy of existing tools. Perhaps most importantly, the absence of benchmarks thwarts debate over TE annotation accuracy because there simply is little data to discuss. This lack of debate has the insidious effect that many of the ultimate end-users of TE annotation, researchers in the broader genomics, and genetics community who are not TE experts are left largely unaware of the complexities and pitfalls of TE annotation. These downstream researchers thus often simply ignore the impact of TE annotation quality on their results, leading to potentially avoidable problems, such as failed experiments or invalid conclusions. Thus, the lack of TE annotation benchmarks hinders the progress of not only TE research but also genomics and related fields in general.

At a recent conference at McGill University's Bellairs Research Institute (St. James Parish, Barbados), a group of TE annotation and tools experts, including the authors, met to discuss these issues. We identified, as a cornerstone of future improvements to computational TE identification systems, a pressing need to create and to widely adopt benchmarks to measure the accuracy of TE annotation methods and tools and to facilitate meaningful comparisons between them. To clarify, we propose to generate benchmarks for genomic TE annotations, not intermediate steps such as library creation, although the latter would also be interesting to benchmark eventually. Benchmark creation will help alleviate all of the aforementioned issues. It will enable tool users to choose the best available tool(s) for their studies and to produce more accurate results, and it will democratize access, encouraging tool creation by additional researchers, particularly those with limited resources. Establishing benchmarks might also encourage the development of experimental pipelines to validate computational TE predictions. Perhaps most importantly, the adoption of standard benchmarks will increase transparency and accessibility, stimulating debate and leading the broader genomics-related research community towards an improved understanding of TEs and TE annotation. Thus, creating benchmarks may lead not only to improved annotation accuracy but may help to demystify a critical area of research that, relative to its importance, is often neglected and misinterpreted. We therefore believe that the TE research community should resolve to agree upon, create, and adopt standard sets of TE annotation benchmarks.

\section{What might TE annotation benchmarks consist of?}

One of the reasons the TE annotation community still does not have accepted benchmarks may be that creating them is more challenging than in other fields. There are many possibilities for the form of such benchmarks and how they could be created. Ideally, they would consist of diverse, perfectly annotated, real genomic sequences; however, irrespective of the efforts made, a perfect TE annotation is impossible to achieve because it is irrevocably based on and limited by current TE detection methods. For instance, greatly decayed and rare TEs are difficult to detect and thus are sources of false negatives. Furthermore, highly heterogeneous TEs can be difficult to accurately assign to families, especially when they are decayed. To illustrate the potential extent of the first of these sources, it is likely that much of the unannotated part (about $40 \%$ ) of the human genome is comprised of ancient TE relics that are too diverged from each other to be currently recognized as such $[1,2,8$, $62,63]$. At a smaller scale, low copy-number TEs are missed by methods that rely on repetitiveness, including most tools used for building repeat libraries, but could be (originally) detected by structural signatures or by approaches using comparative genomics or other genomic attributes. An example of problematic TEs with illdefined and highly heterogeneous structure is the helitron superfamily. Helitrons were initially discovered by computational analysis, based on the repetitiveness of some helitron families and the presence of genes and structural features not found in other TEs [64]. Although some families in some genomes can be detected through repetitiveness, in general, helitrons are especially difficult to detect because they do not have strong structural signatures, are often quite large, lack "canonical" TE genes, and conversely often do contain segments of low copy-number, non-TE (transduplicated) genome sequence [65-67]. Yet in many species, helitrons represent one of the most frequent types of TEs in the genome [64, 68-70]. In general, such false negatives in annotated real genomic data are a problem for benchmarking, since tools that manage to detect true TEs missing from the benchmark would be wrongly penalized. Conversely, false positives present in the benchmark would penalize tools with improved specificity. Ideally, the benchmarks would provide support for probabilistic annotations in order to help account for such uncertainties.

To overcome such issues with annotated genomic sequences, various approaches can be used. False negatives can be predicted by placing fragments of known TEs into real or synthetic genomes, an approach that is especially important for fragmented and degraded TEs [2]. False negatives caused by TE degradation can also be predicted using real genome sequences with known TEs that have been modified in silico by context sensitive evolutionary models [71]. False positive prediction is perhaps a more difficult problem. Because we do not have real genomic regions that we are certain have not been derived from TEs, a variety of methods have been 
used to produce false-positive benchmarks in which no true TE instances are expected to be found. These include reversing (but not complementing) real genomic sequence $[3,72]$ (which is also useful for detecting false extensions, i.e., predicted boundaries that extend beyond actual TEs [73]), shuffling real sequence while preserving mono- or di-nucleotide frequencies [2], and generating sequence using higher-order models [74]. Higher-order models may incorporate multiple key aspects of genome composition, complexity, and repeats, such as the diversity of TEs and their insertion patterns, the distribution of simple repeats and GC-content (compositional domains), varying rates of TE deletion, and other evolutionary processes [75]. Finally, it is important in any of these analyses to distinguish false positives (sequences that may have been generated by chance from mutation processes) from mis-annotation (sequences derived from other repetitive sequence or other TEs than the one being considered).

Even greater challenges are to predict mis-annotation or compound annotation of gene-like sequences that may be derived from TEs, as well as low complexity regions (e.g., CpG islands, pyrimidine stretches, and ATrich regions) [74]. Another serious challenge is to avoid creating biases either for or against the methods used to initially identify any TEs incorporated into the models; for instance, if a certain tool originally identified a TE sequence, then that tool may have an advantage in accurately (re-) identifying the TE in a simulated genome. Furthermore, simulated genomes are not currently useful in evaluating TE annotation methods that employ additional types of data that are impractical to simulate, such as comparative genomic data or realistic populations of small RNA sequences. Finally and most fundamentally, the unknown cannot be modeled, and much about TE sequences, how they transpose, and how they evolve remains unknown. We need to consider, for example, how much our techniques are biased towards the types of TEs present in taxa that we have studied most intensively (e.g., mammals) and against TEs that have evolved in under-represented genomes. Thus, in designing and using standard benchmarks, we must remain cognizant that while improving our ability to detect and annotate TEs, they will also be ultimately limited by current knowledge of TEs and genome evolution.

Although this article is intended to promote discussion rather than providing ultimate solutions, we believe that an ideal benchmark data set would be as follows:

- Contributed, vetted, and periodically revised by the TE annotation community;

- Publicly available;

- A mixture of different types of simulated sequences and well-annotated real genomic regions;
- Sufficiently large in size to allow accurate assessment of tool performance;

- Representative of the biological diversity of genomes (e.g., size, TE density and family representation, evolutionary rates, and GC-content);

- Representative of the various states of assembly of ongoing genome sequencing projects;

- Accompanied by open-source support software that provides both online methods and an application programming interface (API) to compute a range of detailed meaningful statistics on the agreement between a user's annotation and the benchmark data set;

- Eventually, provide support for probabilistic annotations that represent uncertainties, both at the level of the benchmark itself and user submitted annotations.

\section{Why and how should researchers contribute?}

The success of this effort depends on buy-in from the TE community to create and contribute benchmark data sets, to use them in their own work, and to promote their adoption. Because of the multiple challenges involved in the creation of these benchmarks, it is unlikely that any first version will be completely satisfactory; however, this should not be used as an argument to dismiss this type of effort but rather to contribute to its improvement. In the coming months, we would like to initiate discussions with the wider TE community on the ideal format of a first set of TE benchmarks and to begin collecting data sets. We invite the entire TE research community to join us in this effort by providing feedback on the issues raised in this article, by commenting on specific benchmark data set proposals as they are made available, and by contributing their own benchmark data set proposals. To do so, please visit the project's website at http:// cgl.cs.mcgill.ca/transposable-element-benchmarking, or contact the authors.

\section{Abbreviations \\ API: application programming interface; LTR: long terminal repeat; TE: transposable element or DNA originating from them.}

\section{Competing interests}

The authors declare that they have no competing interests.

\section{Authors' contributions}

$\mathrm{MB}$ - with the help of $\mathrm{TB}, \mathrm{DH}$, and $\mathrm{GH}$ - conceived of the topic and organized the conference. All authors $(\mathrm{DH}, \mathrm{GH}, \mathrm{GB}, \mathrm{JC}, \mathrm{RC}, \mathrm{CF}, \mathrm{AF}, \mathrm{AH}, \mathrm{RH}, \mathrm{AK}$, $E L, F M, D P, H Q, A S, T W, T B$, and MB) participated in discussions leading to the ideas presented. DH wrote the manuscript. FM and EL provided data for the figure. All authors read, revised, and approved the final version of the manuscript.

\section{Acknowledgements}

This work was funded by a Bioinformatics and Computational Biology grant to MB and TB from Genome Canada, Genome Québec, and the Canadian Institutes for Health Research. 


\section{Author details}

${ }^{1}$ School of Computer Science, McGill University, McConnell Engineering Bldg., Rm. 318, 3480 Rue University, Montréal, Québec H3A 0E9, Canada. Department of Biology, McGill University, Stewart Biology Bldg., 1205 Ave. du Docteur-Penfield, Montréal, Québec H3A 1B1, Canada. ${ }^{3}$ McGill Centre for Bioinformatics, McGill University, Montréal, Québec, Canada. ${ }^{4}$ Department of Human Genetics, McGill University, Montréal, Québec, Canada. ${ }^{5}$ McGill University and Génome Québec Innovation Center, Montréal, Québec, Canada. ${ }^{6}$ Centre for Research in Agricultural Genomics CSIC-IRTA-UAB-UB, 08193 Barcelona, Spain. ${ }^{7}$ Université de Poitiers, UMR CNRS 7267 Ecologie et Biologie des Interactions, Equipe Ecologie Evolution Symbiose, 5 Rue Albert Turpin, 86073 Poitiers Cedex 9, France. ${ }^{8}$ Department of Human Genetics, University of Utah School of Medicine, Salt Lake City, UT 84112, USA. ${ }^{9}$ Institut des Sciences de l'Evolution de Montpellier (ISE-M), Equipe Evolution, Vecteurs, Adaptation et Symbiose, UMR5554 CNRS-Université Montpellier, Montpellier 34090, cedex 05, France. ${ }^{10}$ Laboratoire Evolution, Génomes, Comportement Ecologie, CNRS_Université Paris-Sud (UMR 9191)—IRD (UMR 247)_Université Paris-Saclay, F-91198 Gif-sur-Yvette, France. ${ }^{11}$ Institute for Systems Biology, 401 Terry Ave. N, Seattle, WA 98109, USA. ${ }^{12}$ Laboratoire Biometrie et Biologie Evolutive, Universite Claude Bernard-Lyon 1, UMR-CNRS 5558-Bat. Mendel, 43 bd du 11 novembre 1918, 69622 Villeurbanne cedex, France. ${ }^{13}$ INRA, UR1164 URGI-Research Unit in Genomics-Info, INRA de Versailles-Grignon, Route de Saint-Cyr, Versailles 78026, France. ${ }^{14}$ University of Colorado School of Medicine, Aurora, CO 80045, USA. ${ }^{15}$ Department of Computer Science, University of Montana, Missoula, MT 59812, USA.

\section{Received: 25 June 2015 Accepted: 22 July 2015}

Published online: 04 August 2015

\section{References}

1. Lander ES, Linton LM, Birren B, Nusbaum C, Zody MC, Baldwin J, et al. Initial sequencing and analysis of the human genome. Nature. 2001;409:860-921.

2. de Koning APJ, Gu W, Castoe TA, Batzer MA, Pollock DD. Repetitive elements may comprise over two-thirds of the human genome. PLoS Genet. 2011;7, e1002384.

3. Wheeler TJ, Clements J, Eddy SR, Hubley R, Jones TA, Jurka J, et al. Dfam: a database of repetitive DNA based on profile hidden Markov models. Nucleic Acids Res. 2013;41(Database issue):D70-82.

4. Schnable PS, Ware D, Fulton RS, Stein JC, Wei F, Pasternak S, et al. The B73 maize genome: complexity, diversity, and dynamics. Science. 2009:326:1112-1115.

5. Solyom S, Kazazian HH. Mobile elements in the human genome: implications for disease. Genome Med. 2012;4:12.

6. Kazazian HH. Mobile elements: drivers of genome evolution. Science. 2004:303:1626-32.

7. Cordaux R, Batzer MA. The impact of retrotransposons on human genome evolution. Nat Rev Genet. 2009;10:691-703.

8. Maumus F, Quesneville H. Deep investigation of Arabidopsis thaliana junk DNA reveals a continuum between repetitive elements and genomic dark matter. PLoS One. 2014:9, e94101.

9. Gifford WD, Pfaff SL, Macfarlan TS. Transposable elements as genetic regulatory substrates in early development. Trends Cell Biol. 2013; doi:10.1016/j.tcb.2013.01.001

10. Lisch DR, Bennetzen JL. Transposable element origins of epigenetic gene regulation. Curr Opin Plant Biol. 2011;14:156-61.

11. Hoen DR, Bureau TE. in Plant transposable elements. Springer Berlin Heidelberg; 2012. 24, p. 219-251.

12. Volff J-N. Turning junk into gold: domestication of transposable elements and the creation of new genes in eukaryotes. Bioessays. 2006;28:913-22.

13. Hoen DR, Bureau TE. Discovery of novel genes derived from transposable elements using integrative genomic analysis. Mol Biol Evol. 2015;32:1487-1506.

14. Li Y, Li C, Xia J, Jin Y. Domestication of transposable elements into MicroRNA genes in plants. PLOS One. 2011;6, e19212.

15. Kelley D, Rinn J. Transposable elements reveal a stem cell-specific class of long noncoding RNAs. Genome Biol. 2012;13:R107.

16. Kapusta A, Kronenberg Z, Lynch VJ, Zhuo X, Ramsay L, Bourque G, et al. Transposable elements Are major contributors to the origin, diversification, and regulation of vertebrate long noncoding RNAs. PLoS Genet. 2013;9:e1003470.
17. Jacques P-É, Jeyakani J, Bourque G. The majority of primate-specific regulatory sequences are derived from transposable elements. PLoS Genet. 2013;9, e1003504.

18. Sundaram V, Cheng Y, Ma Z, Li D, Xing X, Edge $P$, et al. Widespread contribution of transposable elements to the innovation of gene regulatory networks. Genome Res. 2014;24:1963-76.

19. Wicker T, Sabot F, Hua-Van A, Bennetzen JL, Capy P, Chalhoub B, et al. A unified classification system for eukaryotic transposable elements. Nat Rev Genet. 2007:8:973-82.

20. Kapitonov W, Jurka J. A universal classification of eukaryotic transposable elements implemented in Repbase. Nat Rev Genet. 2008;9:411-2. author reply 414

21. Quesneville H, Bergman CM, Andrieu O, Autard D, Nouaud D, Ashburner M, et al. Combined evidence annotation of transposable elements in genome sequences. PLoS Comput Biol. 2005;1:166-75.

22. Hu T, Pattyn P, Bakker EG, Cao J, Cheng J-F, Clark RM, et al. The Arabidopsis lyrata genome sequence and the basis of rapid genome size change. Nat Genet. 2011;43:476-81.

23. Bergman CM, Quesneville $\mathrm{H}$. Discovering and detecting transposable elements in genome sequences. Brief Bioinformatics. 2007:8:382-92.

24. Lerat E. Identifying repeats and transposable elements in sequenced genomes: how to find your way through the dense forest of programs. Heredity. 2010;104:520-33.

25. Flutre T, Permal E, Quesneville H. in Plant transposable elements. Springer Berlin Heidelberg; 2012. 24, p. 17-39.

26. Saha S, Bridges S, Magbanua ZV, Peterson DG. Computational approaches and tools used in identification of dispersed repetitive DNA sequences. Tropical Plant Biol. 2008;1:85-96.

27. Caspi A, Pachter L. Identification of transposable elements using multiple alignments of related genomes. Genome Res. 2006;16:260-70.

28. El-Baidouri M, Kim KD, Abernathy B, Arikit S, Maumus F, Panaud O, et al. A new approach for annotation of transposable elements using small RNA mapping. Nucleic Acids Res. 2015;gkv257.

29. Benson G. Tandem repeats finder: a program to analyze DNA sequences. Nucleic Acids Res. 1999;27:573-80.

30. Smit A, Hubley R. RepeatModeler Open-1.0. Repeat Masker Website (2010) at <http://www.repeatmasker.org >.

31. Bao Z, Eddy SR. Automated de novo identification of repeat sequence families in sequenced genomes. Genome Res. 2002;12:1269-76.

32. Price $\mathrm{AL}$, Jones NC, Pevzner PA. De novo identification of repeat families in large genomes. Bioinformatics. 2005;21 Suppl 1:i351-8.

33. Xu Z, Wang H. LTR_FINDER: an efficient tool for the prediction of full-length LTR retrotransposons. Nucleic Acids Res. 2007;35:W265-8.

34. McCarthy EM, MCDonald JF. LTR_STRUC: a novel search and identification program for LTR retrotransposons. Bioinformatics. 2003;19:362-7.

35. Han $Y$, Wessler SR. MITE-Hunter: a program for discovering miniature inverted-repeat transposable elements from genomic sequences. Nucleic Acids Res. 2010;38:e199-9.

36. Feschotte C, Keswani U, Ranganathan N, Guibotsy ML, Levine D. Exploring repetitive DNA landscapes using REPCLASS, a tool that automates the classification of transposable elements in eukaryotic genomes. Genome Biol Evol. 2009;1:205-20.

37. Jurka J, Kapitonov W, Pavlicek A, Klonowski P, Kohany O, Walichiewicz J. Repbase update, a database of eukaryotic repetitive elements. Cytogenet Genome Res. 2005:110:462-7.

38. Smit A, Hubley R, Green P. 1996-2010. RepeatMasker Open-3.0. at $<$ http://www.repeatmasker.org >.

39. Kohany O, Gentles AJ, Hankus L, Jurka J. Annotation, submission and screening of repetitive elements in Repbase: RepbaseSubmitter and Censor. BMC Bioinformatics. 2006;7:474.

40. Green P. Cross_match. at <http://www.phrap.org/phredphrapconsed.html>

41. Altschul SF, Gish W, Miller W, Myers EW, Lipman DJ. Basic local alignment search tool. J Mol Biol. 1990;215:403-10.

42. Camacho C, Coulouris G, Avagyan V, Ma N, Papadopoulos J, Bealer K, et al. BLAST+: architecture and applications. BMC Bioinformatics. 2009;10(1):421. http://doi.org/10.1186/1471-2105-10-421

43. Wheeler TJ, Eddy SR. nhmmer: DNA homology search with profile HMMs. Bioinformatics. 2013;29:2487-9.

44. Flutre T, Duprat E, Feuillet C, Quesneville H. Considering transposable element diversification in de novo annotation approaches. PLoS One. $2011 ; 6$, e 16526. 
45. Li R, Ye J, Li S, Wang J, Han Y, Ye C, et al. ReAS: recovery of ancestral sequences for transposable elements from the unassembled reads of a whole genome shotgun. PLoS Comput Biol. 2005;1:313-21.

46. DeBarry JD, Liu R, Bennetzen JL. Discovery and assembly of repeat family pseudomolecules from sparse genomic sequence data using the assisted automated assembler of repeat families (AAARF) algorithm. BMC Bioinformatics. 2008;9:235.

47. Keane TM, Wong K, Adams DJ. RetroSeq: transposable element discovery from next-generation sequencing data. Bioinformatics. 2013;29:389-90.

48. Zytnicki M, Akhunov E, Quesneville H. Tedna: a transposable element de novo assembler. Bioinformatics. 2014;30:2656-8.

49. Koch P, Platzer M, Downie BR. RepARK—de novo creation of repeat libraries from whole-genome NGS reads. Nucleic Acids Res. 2014;42:gku210-e80.

50. Fiston-Lavier A-S, Barrón MG, Petrov DA, González J. T-lex2: genotyping, frequency estimation and re-annotation of transposable elements using single or pooled next-generation sequencing data. Nucleic Acids Res. 2015;43:e22-2.

51. Ouyang S, Buell C. The TIGR plant repeat databases: a collective resource for the identification of repetitive sequences in plants. Nucleic Acids Res. 2004;32:D360-3.

52. Saha S, Bridges S, Magbanua ZV, Peterson DG. Empirical comparison of ab initio repeat finding programs. Nucleic Acids Res. 2008;36:2284-94.

53. Ragupathy R, You FM, Cloutier S. Arguments for standardizing transposable element annotation in plant genomes. Trends Plant Sci. 2013; doi:10.1016/ j.tplants.2013.03.005.

54. Bradnam KR, Fass JN, Alexandrov A, Baranay P, Bechner M, Birol I, et al. Assemblathon 2: evaluating de novo methods of genome assembly in three vertebrate species. Gigascience. 2013;2:10.

55. Balaji S, Sujatha S, Kumar SS, Srinivasan N. PALI - a database of Phylogeny and ALlgnment of homologous protein structures. Nucleic Acids Res. 2001;29:61-5.

56. Van Walle I, Lasters I, Wyns L. SABmark-a benchmark for sequence alignment that covers the entire known fold space. Bioinformatics. 2005;21:1267-8.

57. Thompson JD, Koehl P, Ripp R, Poch O. BAliBASE 3.0: latest developments of the multiple sequence alignment benchmark. Proteins. 2005;61:127-36.

58. Talwalkar A, Liptrap J, Newcomb J, Hartl C, Terhorst J, Curtis K, et al. SMaSH: a benchmarking toolkit for human genome variant calling. Bioinformatics. 2014;30:2787-2795

59. Kim SY, Speed TP. Comparing somatic mutation-callers: beyond Venn diagrams. BMC Bioinformatics. 2013;14:189.

60. Boutros PC, Margolin AA, Stuart JM, Califano A, Stolovitzky G. Toward better benchmarking: challenge-based methods assessment in cancer genomics. Genome Biol. 2014;15:462.

61. Moult J. A decade of CASP: progress, bottlenecks and prognosis in protein structure prediction. Curr Opin Struct Biol. 2005;15:285-9.

62. Doolittle WF, Sapienza C. Selfish genes, the phenotype paradigm and genome evolution. Nature. 1980;284:601-3.

63. Smit AF. The origin of interspersed repeats in the human genome. Curr Opin Genet Dev. 1996;6:743-8.

64. Kapitonov W, Jurka J. Rolling-circle transposons in eukaryotes. Proc Natl Acad Sci U S A. 2001;98:8714-9

65. Jiang N, Bao Z, Zhang X, Eddy SR, Wessler SR. Pack-MULE transposable elements mediate gene evolution in plants. Nature. 2004;431:569-73.

66. Juretic N, Hoen DR, Huynh ML, Harrison PM, Bureau TE. The evolutionary fate of MULE-mediated duplications of host gene fragments in rice. Genome Res. 2005;15:1292-7.

67. Morgante M, Brunner S, Pea G, Fengler K, Zuccolo A, Rafalski A. Gene duplication and exon shuffling by helitron-like transposons generate intraspecies diversity in maize. Nat Genet. 2005;37:997-1002.

68. Pritham EJ, Feschotte C. Massive amplification of rolling-circle transposons in the lineage of the bat Myotis lucifugus. Proc Natl Acad Sci U S A. 2007;104:1895-900

69. Yang L, Bennetzen JL. Distribution, diversity, evolution, and survival of Helitrons in the maize genome. Proc Natl Acad Sci U S A. 2009;106:19922-7.

70. Thomas J, Vadnagara K, Pritham. DINE-1, the highest copy number repeats in Drosophila melanogaster are non-autonomous endonuclease-encoding rolling-circle transposable elements (helentrons). Mob DNA. 2014;5:18.

71. Edgar RC, Asimenos G, Batzoglou S, Sidow A. Evolver. at $<$ http://www.drive5.com/evolver>
72. Schwartz S, Kent WJ, Smit A, Zhang Z, Baertsch R, Hardison RC, et al. Human-mouse alignments with BLASTZ. Genome Res. 2003;13(1):103-7. http://doi.org/10.1101/gr.809403.

73. Frith MC, Park Y, Sheetlin SL, Spouge JL. The whole alignment and nothing but the alignment: the problem of spurious alignment flanks. Nucleic Acids Res. 2008;36:5863-71.

74. Caballero J, Smit AFA, Hood L, Glusman G. Realistic artificial DNA sequences as negative controls for computational genomics. Nucleic Acids Res. 2014;42:e99-9

75. Levin HL, Moran JV. Dynamic interactions between transposable elements and their hosts. Nat Rev Genet. 2011;12:615-27.

76. Kent WJ, Sugnet CW, Furey TS, Roskin KM, Pringle TH, Zahler AM, et al. The human genome browser at UCSC. Genome Res. 2002;12(6):996-1006. http://doi.org/10.1101/gr.229102

77. Haudry A, Platts AE, Vello E, Hoen DR, Leclercq M, Williamson RJ, et al. An atlas of over 90,000 conserved noncoding sequences provides insight into crucifer regulatory regions. Nat Genet. 2013;45:891-8.

78. de-la-Chaux N, Tsuchimatsu T, Shimizu KK, Wagner A. The predominantly selfing plant Arabidopsis thaliana experienced a recent reduction in transposable element abundance compared to its outcrossing relative Arabidopsis lyrata. Mob DNA. 2012;3:2.

79. Ellinghaus D, Kurtz S, Willhoeft U. LTRharvest, an efficient and flexible software for de novo detection of LTR retrotransposons. BMC Bioinformatics. 2008;9:18.

80. Salamov AA, Solovyev W. Ab initio gene finding in Drosophila genomic DNA. Genome Res. 2000;10:516-22.

81. Cai J, Liu X, Vanneste K, Proost S, Tsai W-C, Liu K-W, et al. The genome sequence of the orchid Phalaenopsis equestris. Nat Genet. 2015;47(1):65-72. http://doi.org/10.1038/ng.3149.

82. Xu P, Zhang X, Wang X, Li J, Liu G, Kuang Y, et al. Genome sequence and genetic diversity of the common carp, Cyprinus carpio. Nat Genet. 2014;46:1212-9. http://doi.org/10.1038/ng.3098.

83. Rondeau EB, Minkley DR, Leong JS, Messmer AM, Jantzen JR, Schalburg von $K R$, et al. The genome and linkage map of the northern pike (Esox lucius): conserved synteny revealed between the salmonid sister group and the Neoteleostei. PLoS One. 2014;9(7), e102089. http://doi.org/10.1371/ journal.pone.01020.

84. Wang M, Yu Y, Haberer G, Marri PR, Fan C, Goicoechea JL, et al. The genome sequence of African rice (Oryza glaberrima) and evidence for independent domestication. Nat Genet. 2014;46(9):982-8. http://doi.org/ 10.1038/ng.3044

85. Marmoset Genome Sequencing and Analysis Consortium. The common marmoset genome provides insight into primate biology and evolution. Nat Genet. 2014;46:850-7.

86. Li F, Fan G, Wang K, Sun F, Yuan Y, Song G, et al. Genome sequence of the cultivated cotton Gossypium arboreum. Nat Genet. 2014;46(6):567-72. http://doi.org/10.1038/ng.2987.

87. Sierro N, Battey JND, Ouadi S, Bakaher N, Bovet L, Willig A, et al. The tobacco genome sequence and its comparison with those of tomato and potato. Nat Commun. 2014;5:3833. http://doi.org/10.1038/ncomms4833.

88. International Glossina Genome Initiative. Genome sequence of the tsetse fly (Glossina morsitans): vector of African trypanosomiasis. Science. 2014;344:380-386.

89. Berthelot C, Brunet F, Chalopin D, Juanchich A, Bernard M, Noel B, et al. The rainbow trout genome provides novel insights into evolution after whole-genome duplication in vertebrates. Nat Commun. 2014;5:3657. http://doi.org/10.1038/ncomms4657.

90. Wang B, Ekblom R, Bunikis I, Siitari H, Höglund J. Whole genome sequencing of the black grouse (Tetrao tetrix): reference guided assembly suggests faster-Z and MHC evolution. BMC Genomics. 2014;15:180

91. Wegrzyn JL, Liechty JD, Stevens KA, Wu L-S, Loopstra CA, Vasquez-Gross HA, et al. Unique features of the loblolly pine (Pinus taeda L.) megagenome revealed through sequence annotation. Genetics. 2014;196(3):891-909. http://doi.org/10.1534/genetics.113.159996.

92. Wang W, Haberer G, Gundlach H, Gläßer C, Nussbaumer T, Luo MC, et al The Spirodela polyrhiza genome reveals insights into its neotenous reduction fast growth and aquatic lifestyle. Nat Commun. 2014;5. http://doi.org/10.1038/ncomms4311.

93. Chen S, Zhang G, Shao C, Huang Q, Liu G, Zhang P, et al. Whole-genome sequence of a flatfish provides insights into ZW sex chromosome evolution and adaptation to a benthic lifestyle. Nat Genet. 2014;46(3):253-60. http://doi.org/10.1038/ng.2890. 
94. Qin C, Yu C, Shen Y, Fang X, Chen L, Min J, et al. Whole-genome sequencing of cultivated and wild peppers provides insights into Capsicum domestication and specialization. Pnas. 2014;111(14):5135-40. http://doi.org/ 10.1073/pnas. 1400975111

95. Kim S, Park M, Yeom S-I, Kim Y-M, Lee JM, Lee H-A, et al. Genome sequence of the hot pepper provides insights into the evolution of pungency in Capsicum species. Nat Genet. 2014;46(3):270-8. http://doi.org/10.1038/ ng.2877.

96. Zhou D, Zhang D, Ding G, Shi L, Hou Q, Ye Y, et al. Genome sequence of Anopheles sinensis provides insight into genetics basis of mosquito competence for malaria parasites. BMC Genomics. 2014;15(1):42. http://doi.org/10.1186/1471-2164-15-42.

\section{Submit your next manuscript to BioMed Central} and take full advantage of:

- Convenient online submission

- Thorough peer review

- No space constraints or color figure charges

- Immediate publication on acceptance

- Inclusion in PubMed, CAS, Scopus and Google Scholar

- Research which is freely available for redistribution 